\title{
Enhancing the Kerr nonlinearity in SiN nanowires with graphene oxide films
}

\author{
Yang Qu ${ }^{\mathrm{a}}$, Jiayang $\mathrm{Wu}^{\mathrm{a}}$, Yunyi Yanga, Yuning Zhang ${ }^{\mathrm{a}}$, Houssein El Dirani ${ }^{\mathrm{b}}$, Romain Crochemore ${ }^{\mathrm{b}}$, \\ Corrado Sciancalepore $^{\mathrm{b}}$, Pierre Demongodin ${ }^{\mathrm{c}}$, Christelle Monat ${ }^{\mathrm{c}}$, Baohua Jia, ${ }^{\mathrm{d}}, *$ and David J. Moss ${ }^{\mathrm{a}}$ * \\ ${ }^{a}$ Optical Sciences Centre, Swinburne University of Technology, Hawthorn, VIC 3122, Australia \\ ${ }^{b}$ University Grenoble Alpes, CEA-LETI, Minatec, Optics ans Photonics Division, 17 rue des Martyrs, 38054 Grenoble, France \\ ${ }^{c}$ Institut des nanotechnologies de Lyon, UMR CNRS 5270, Ecole Centrale Lyon, F-69130 Ecully, France \\ ${ }^{d}$ Centre for Translational Atomaterials, Swinburne University of Technology, Hawthorn, VIC 3122, Australia
}

Abstract: Enhanced four-wave mixing in silicon nitride waveguides integrated with 2D graphene oxide (GO) films is experimentally demonstrated. We achieve a high conversion efficiency improvement of $\sim 7.3 \mathrm{~dB}$ for a 2-cm-long waveguide with monolayer $\mathrm{GO}$ film.

\section{Introduction}

Four wave mixing (FWM), a fundamental third-order nonlinear optical process [1-3], has been widely applied to all-optical signal generation and processing [4,5]. Efficient FWM has been demonstrated in III-V platforms such as GaAs and AlGaAs [4], and in CMOS (Complementary Metal Oxide Semiconductor) compatible platforms including silicon, silicon nitride, and high index doped silica glass [5, 6]. Despite silicon being a leading platform for integrated photonic devices, its strong two-photon absorption (TPA) in the telecom band near $1550 \mathrm{~nm}$ significantly limits its nonlinear performance [5]. Other CMOS compatible platforms such as silicon nitride and high index doped silica have much weaker TPA, although they face limitations in terms of FWM efficiency since their Kerr nonlinearity $\left(n_{2}\right)$ is over an order of magnitude lower than silicon [6]. The quest for high-performance nonlinear integrated photonic devices has motivated the use of highly nonlinear materials on chips to overcome the limitations of existing platforms.

Recently, the giant Kerr nonlinear response of two-dimensional (2D) materials such as graphene, graphene oxide (GO), black phosphorus, and transition metal dichalcogenides (TMDCs) has been widely recognized and exploited to implement diverse nonlinear photonic devices with high performance and new capabilities [7-9]. Due to its ease of preparation as well as the tunability of its material properties, GO has become a highly promising member of the 2D material family [9]. Recently, we reported GO films with a giant Kerr nonlinear response about 4 orders of magnitude higher than that of silicon and demonstrated enhanced four-wave mixing (FWM) in doped silica waveguides and MRRs integrated with GO films [10, 11]. In this paper, we report significantly improved FWM performance for $\mathrm{Si}_{3} \mathrm{~N}_{4}$ waveguides incorporated with $2 \mathrm{D} \mathrm{GO}$ film. Owing to strong mode overlap between the integrated waveguide and the GO films that have a high Kerr nonlinearity and low loss, the FWM efficiency is significantly improved. We perform FWM measurements for different pump powers and numbers of GO layers, achieving up to $7.3 \mathrm{~dB}$ enhancement in the FWM conversion efficiency (CE) for a 2-cmlong waveguide integrated with 1 layer of GO. These results confirm the improved FWM performance of $\mathrm{Si}_{3} \mathrm{~N}_{4}$ waveguides incorporating 2D GO films.

\section{Device fabrication and characterization}

Figure 1(a) shows a schematic of the GO-coated $\mathrm{Si}_{3} \mathrm{~N}_{4}$ waveguide, with a cross section of $1.6 \mu \mathrm{m} \times 0.66$ $\mu \mathrm{m}$. The integrated waveguide is surrounded by silica, except that the upper cladding is removed to enable coating the waveguide with GO films. The GO films, with a thickness of $2 \mathrm{~nm}$ per layer, were coated on the top of the integrated waveguide in order to allow for the light-material interaction with the evanescent field leaking from the waveguide. According to our previous measurements [10-12], the Kerr coefficient of GO is on the order of $10^{-15}$ to $10^{-14} \mathrm{~m}^{2} / \mathrm{W}$, which is slightly lower than that of graphene $\left(\sim 10^{-13} \mathrm{~m}^{2} / \mathrm{W}\right)$ [12], but still orders of magnitude higher than $\mathrm{Si}_{3} \mathrm{~N}_{4}\left(\sim 10^{-19} \mathrm{~m}^{2} / \mathrm{W}\right)$. An image of the integrated waveguide incorporating 10 layers of GO is shown in Fig. 1(b), which illustrates that the morphology is of high quality, leading to a high transmittance of the GO film on top of the integrated waveguide. Figure 1(c) shows a scanning electron microscope (SEM) image of a 2D layered GO film on a silica substrate, with up to 5 layers of GO.
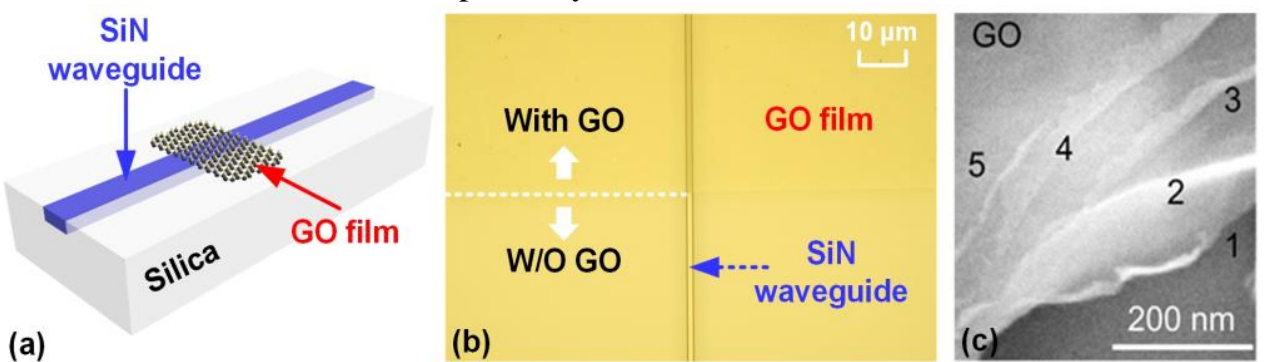

Fig. 1. (a) Schematic illustration of hybrid waveguides integrated with GO. (b)Image of hybrid integrated waveguide with 10 layers of GO. (c) SEM image of the five-layer GO structure 

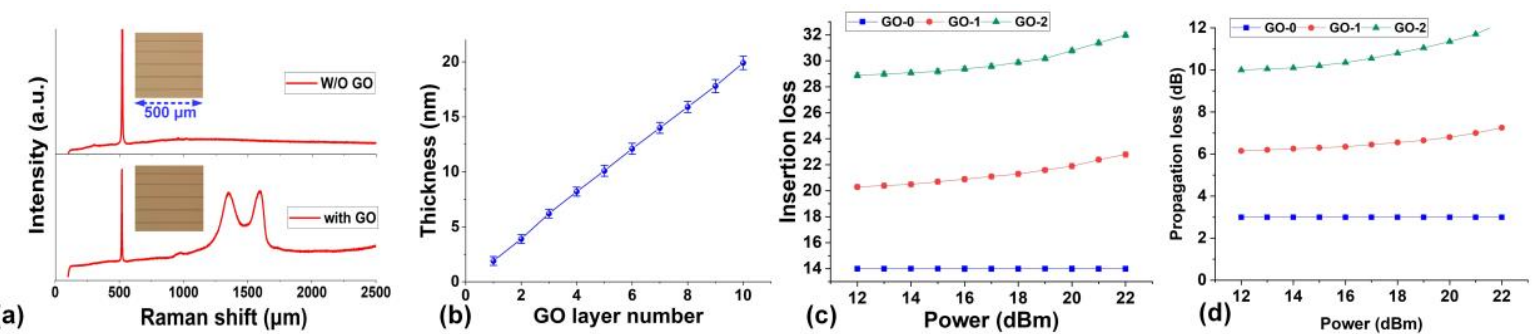

Fig. 2. (a) Raman spectra of GO on the integrated SiN chip. (b) Thickness of GO films versus the layer number. (c) Insertion loss of the hybrid waveguide with 0,1 and 2 layers of GO films under different input coupling power. (d) Propagation loss of the integrated waveguides with 0,1 and 2 layers of GO under different input power.

The integration of GO onto the waveguide is confirmed by Raman spectra in Fig. 2 (a) with the representative D and G peaks of GO. Figure 2(b) shows the thickness of GO films versus the layer number characterized by atomic force microscopy. The total insertion loss of the integrated waveguides with different numbers of GO films under different input powers is depicted in Fig. 2 (c). The propagation loss of the bare waveguide and the waveguide with a monolayer of GO was $\sim 3 \mathrm{~dB} / \mathrm{cm}$ and $\sim 6 \mathrm{~dB} / \mathrm{cm}$, respectively, which are shown in Fig. 2 (d), corresponding to an excess propagation loss of $\sim 3 \mathrm{~dB} / \mathrm{cm} /$ layer induced by the $\mathrm{GO}$ film. This is about 2 orders of magnitude lower than silicon waveguides coated with graphene [13]. The insertion loss slightly increases with the input CW power, which is mainly induced by photo-thermal effects of the GO films at higher powers [14-16].

\section{FWM experiments}

We used the experimental setup shown in Fig. 3(a) to perform FWM measurements in the GO hybrid integrated waveguides. The FWM spectra of a 2 -cm-long integrated waveguide without GO and with 1 layer and 2 layers of GO are shown in Fig. 3(b). For comparison, we kept the same pump power of $\sim 26 \mathrm{dBm}$ before the input of the waveguide, which corresponded to $\sim 21 \mathrm{dBm}$ pump power coupled into the waveguide. It can be seen that although the hybrid integrated waveguide had an additional propagation loss of $\sim 7.4 \mathrm{~dB}$, it clearly shows an enhanced $\mathrm{CE}$ of $7.3 \mathrm{~dB}$ as compared with the same waveguide without GO. The CE for various pump powers coupled to the waveguide without GO and with 1 layer and 2 layers of GO are shown in Fig. 3(c). One can see that as the pump power increased, the $\mathrm{CE}$ increased with no obvious saturation for all samples, which reflects the low nonlinear absorption of both the $\mathrm{Si}_{3} \mathrm{~N}_{4}$ and the GO layers in the telecommunications band. These hybrid integrated devices offer a powerful solution to implement high performance nonlinear photonic devices, thus holding great promise for future ultra-high-speed all-optical information processing.

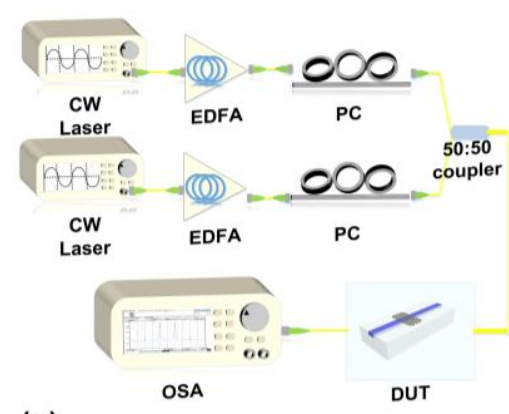

(a)

Fig. 3. (a) Experimental setup for the testing FWM in the GO hybrid integrated waveguide. EDFA: Erbrium-doped fibre amplifier, PC: polarization controller, DUT: device under test, and OSA: optical spectrum analyser. (b) FWM spectra of the integrated waveguide without GO and with 1 and 2 layers of GO. (c) Measured CE versus pump powers for the waveguide without GO and with 1 and 2 layers of GO.

\section{References}

[1] D. Moss et al., "THG as a structural diagnostic of ion-implanted amorphous and crystalline Si," App. Phys. Lett, 48 (17), 1150-1152. 1986.

[2] B. Corcoran, et al., "Green light emission in silicon through slow-light enhanced third-harmonic generation in photonic-crystal waveguides," Nature Photonics, vol. 3, no. 4, pp. 206-210. 2009.

[3] D. J. Moss, H. M. van Driel, and J. E. Sipe, "Dispersion in the anisotropy of optical third-harmonic generation in silicon," Opt. Lett., vol. 14, no. 1, pp. 57-59. 1989.

[4] F. Morichetti et al., "Travelling-wave resonant FWM breaks the limits of cavity-enhanced all-optical wavelength conversion," Nat. Commun, 2(1), 296, 2011.

[5] J. Leuthold et al., "Nonlinear silicon photonics," Nat. Photonics, 4(8), 535-544, 2010.

[6] D. Moss et al., "New CMOS-compatible platforms based on SiN and Hydex for nonlinear optics", Nat. Photonics., 7 (8) $597,2013$.

[7] P. Demongodin et al., "Ultrafast saturable absorption dynamics in hybrid graphene/Si3N4 waveguides," APL Photonics, 4(7), 2019.

[8] N. Dong et al., "Saturation of two-photon absorption in layered transition metal dichalcogenides: experiment and theory." ACS Photonics, $\mathbf{5}(4), 1558,2018$.

[9] W. Gao, "The chemistry of graphene oxide", in Graphene oxide: reduction recipes, spectroscopy, and applications, W. Gao, Ed. Cham: Springer International Publishing, 61-95, 2015.

[10] Y. Yang et.al., "Enhanced four-wave mixing in waveguides integrated with graphene oxide," APL Photonics, 3(12), $120803,2018$.

[11] J. Wu et.al., "Enhanced nonlinear FWM in MRRs integrated with layered GO films", Small 16 (16) 1906563 (2020). doi: 10.1002/smll.201906563. 
[12] X. Zheng et al., "In situ third-order nonlinear responses during laser reduction of graphene oxide thin films towards on-chip nonlinear photonic devices," Adv. Mater., 26(17), 2699-2703, 2014.

[13] M. Ji et al., "Enhanced parametric frequency conversion in a compact silicon-graphene microring resonator," Opt. Exp., 23(14) 18679$18685,2015$.

[14] J. Wu et al., "Graphene oxide waveguide and micro-ring resonator polarizers," Laser Photonics Rev., 13(9), 1900056, 2019.

[15] Y. Zhang et al., "Enhanced Kerr nonlinearity and nonlinear figure of merit in silicon nanowires integrated with 2D graphene oxide films", ACS Applied Materials and Interfaces $\underline{12}$ (29) 33094-33103 June 29 (2020). DOI:10.1021/acsami.0c07852

[16] Y.Qu et al., "Enhanced nonlinear four-wave mixing in silicon nitride waveguides integrated with 2D layered graphene oxide films", arXiv:2006.14944 (2020). 